that, unlike diseases such as dengue fever or malaria, but like H1N1, HFMD can be transmitted directly from human to human; no animal reservoir is required. Cases start out with flu-like symptoms that can develop into characteristic sores in the mouth and rashes on the palms and soles of the feet. Infections can also lead to meningitis and swelling of the brain, possibly resulting in long-term neurological damage (L.-Y. Chang et al. N. Engl. J. Med. 356, 1226-1234; 2007) or even death. The vast majority of reported cases and almost all of the serious cases are in patients under 5 years old.

In 2008, more than 500,000 HFMD infections were reported in China, and 200 people died. This year, as the disease moves through its peak season, similar numbers look possible. There is currently no vaccine to prevent it and no drug to cure it. The best safeguard is good hygiene: HFMD transmission is mainly via saliva and faeces. But even Singapore, renowned for its cleanliness, has had 8,896 cases this year, and had had even more by this time last year. There have also been cases this year in Taiwan, Hong Kong and South Korea.

Most of the serious HFMD cases seem to be caused by enterovirus 71 (EV71), first isolated in California in 1969. EV71, in turn, is related to poliovirus - a fact that has some experts concerned. EV71's recent pattern, with a few isolated outbreaks that are building in frequency, is similar to that seen before the twentieth-century polio epidemics that killed or crippled tens of millions of people around the world (J. F. Modlin N. Engl. J. Med. 356, 1204-1205; 2007). Although it is far from certain that EV71 will follow a similar course, the virus does seem to have become increasingly virulent in China since 2004, possibly because of genetic changes (see Nature 458, 554-555; 2009).

Thus the balancing act: China, the WHO and all of the other health organizations are to be commended for mounting a vigorous response to H1N1 (see page 756). But given the ongoing damage being caused by EV71, and its potential for large-scale, person-to-person spread, they should also be mounting a substantial effort to understand and counter that virus. And indeed, the WHO had a meeting on EV71 in Beijing earlier this year.

Yet that good start is not enough. WHO representatives play down the importance of EV71, and there is little information about the virus on the WHO website. One EV71 specialist laments: "All resources seem to be going to influenza." And in the meantime, many doctors in China still misdiagnose HFMD, partly because the virus and its symptoms change, and partly because there are still no good tests to identify it. More leadership, more education, more awareness and much more research on drugs and a vaccine are essential.

\section{The sharing principle}

\section{Journals and funders must insist that genetically modified mouse strains are fully available.}

$\mathrm{B}$ ack in 1996, human-genome scientists signed up to the Bermuda agreement to share their data without delay. Since then, the sharing principle has entered the mainstream - it now applies to all genomic data generated using public funding, as well as to all the relevant resources cited in publications.

But this principle is not universally observed for genetically modified mice, designed as vital resources in the quest to unpick basic biological mechanisms or to model human disease. The size of the problem is unclear, but existing surveys, combined with extensive anecdotal evidence, suggest it is substantial. In April 2006, for example, scientists at the US National Institutes of Health found that nearly 4,000 unique mice strains had been created, yet barely 700 had been placed in a repository.

Some scientists say they do not have the time nor money to breed and distribute their mice, or even to send the animals to publicly funded mouse repositories such as the European Mouse Mutant Archive in Europe, the Jackson Laboratory in Bar Harbor, Maine, and RIKEN BioResource Centre in Ibaraki, Japan, which would do those chores for them. Others claim that the careers of young and vulnerable researchers (or old and vulnerable researchers) could be harmed if they lost their exclusive access to a resource they made for their own research projects. Or, they say their institution's technology-transfer offices or companies will not let them part with mouse strains that could perhaps be made to turn a profit.

Such attitudes were noted with concern last month at a workshop in Rome hosted by CASIMIR, a European Union project to coordinate and sustain mouse resources internationally (see background documents at http://tinyurl.com/l2hyvq). The workshop brought together representatives from funding agencies, publishers and the mouse repositories from Europe, the United States and Australasia. They concluded that the sharing problem urgently needs resolution - not least because international projects to systematically generate mouse lines deficient in each gene in the genome will generate thousands of new strains in the next five years or so.

To solve the problem, however, journals and funding agencies must take a tougher line. The Nature journals are among the very few actually requiring that authors use established public repositories wherever possible as a condition of publication. Most journals simply 'encourage' their authors to make mice used in their publications freely available to other laboratories, or 'suggest' that the mice be deposited in repositories. Funding agencies similarly prefer such cajoling terms as 'encourage' in their policies on sharing mouse resources, and rarely police the outcome.

Journals should now require researchers to place their mice in repositories as a condition of publication. And funding agencies should require repository plans to be included in all grant applications that are likely to generate new mouse strains. Part of the grant money should be reserved for this task and final reports or evaluations of the grants should refer to the repository used. The repositories themselves should help the journals and funding agencies by finding a way to generate a unique accession number for each mouse strain.

The sharing principle allows biology to progress efficiently. It avoids duplication of effort and allows different laboratories to use the same tools. It is essential that scientists sign up to it. Sharing mice has never been easier - the repositories around the world are efficient and professional, and they are coordinated. Just a few changes in the modus operandi of key institutions could ensure that the makers of mice will have no possible excuse not to use them. 